\title{
Late onset of traumatic aortic regurgitation following a blunt chest trauma
}

\begin{abstract}
Aortic valve regurgitation is a rare complication of blunt chest trauma, and when it ocurrs, it usually has a severe and acute onset. We report the case of a 52 year old healthy male who was involved in a high velocity motorcycle accident that caused cranial and thoracic injuries from which he recovered uneventfully after a two week admission in the ICU (Intensive Care Unit); cardiac lesions had been ruled out. Once weaning from mechanical ventilation was attempted the patient presented clear signs of acute heart failure and he was finally diagnosed with an aortic valve rupture that caused massive aortic valve regurgitation. After a surgical replacement of the aortic valve, the patient made a full recovery and was discharged two weeks later.
\end{abstract}

Conclusion: Late presentation of traumatic aortic regurgitation should be considered in patients with a history of chest trauma even if valvular lesions were initially ruled out.

Keywords: aortic valve, traumatic valvular lesion, blunt chest trauma, thoracic trauma
Volume 10 Issue 6 - 2020

\author{
J Gonzalez Londoño, 1,2 C Lorencio \\ Cardenas,' M Morales Fornós, ${ }^{3}$ I Julià Amill, ${ }^{4}$ \\ JM Sirvent ${ }^{1,2}$ \\ 'Intensive Care Unit, Dr Josep Trueta University Hospital, Spain \\ ${ }^{2}$ Intensive Care Unit, Santa Caterina Hospital, Spain \\ ${ }^{3}$ Cardiology Department, Dr JosepTrueta University Hospital, \\ Spain \\ ${ }^{4}$ Cardiac Surgery Department, German Trias i Pujol Hospital, \\ Spain
}

Correspondence: | Gonzalez Londoño, Intensive Care Unit, Dr Josep Trueta University Hospital, Spain, Email jgonzalez.girona.ics@gencat.cat

Received: November 08, 2020 | Published: November 19 , 2020
Abbreviations: ICU, intensive care unit; GCS, glasgow coma scale; TTE, transthoracic echocardiography; TTE, transesophageal echocardiography

\section{Introduction}

Aortic valve regurgitation is a rare complication of blunt chest trauma ${ }^{1-3}$ and usually has a severe and acute presentation. ${ }^{4}$ Focusing on the valvular apparatus sequelae in the post traumatic patient, the lesions that generally occur are: contusion, laceration and rupture. Predicting a clinical presentation or standard anatomical features is impossible because of the variability of the native valve and the mechanism and violence of the trauma. We present the case of a 52 year old healthy male who presented lesions to the aortic valve two weeks after a blunt chest trauma.

\section{Case report}

A 52 year old male was involved in a high velocity motorcycle accident that caused cranial and thoracic injuries. The patient had no medical history of cardiovascular or valvular disease and previous to the accident had been asymptomatic. He was found unconscious, with a Glasgow Coma Scale (GCS) of 6, which required intubation and mechanical ventilation on site. He was transported to the Emergency Department of our Hospital, where upon arrival a Body-CT was performed. There was evidence of a small contusion to the right frontal lobe, fracture of the 2nd-4th right ribs, a right pulmonary contusion and a liver contusion with noactive bleeding. Lesions to the aortic artery were ruled out.

The patient was admitted to the ICU and was handled according to neurotrauma protocol with a good outcome, the liver contusion remained stable and the pulmonary contusion healed adequately. During this period he developed an upper respiratory tract infection, $\mathrm{H}$. influenza was detected a tracheal aspirate and there was an increase of tracheal secretions; there was no evidence of ventilator associated pneumonia at any time. He was treated with Levofloxacin and the follow up cultures were negative. Intracranial pressure remained low and 7 days after the accident the patient began weaning from mechanical ventilation.
Once the weaning process started, the patient presented severe hypoxia that was initially attributed to the respiratory tract infection. He improved gradually and was extubated on day 15 , but required reintubation 12 hours later because of hypoxic respiratory insufficiency. At this point a transthoracic echocardiography (TTE) was performed, valvulopathies were ruled out.

A percutaneous tracheostomy was performed and once again it was attempted to wean the patient from mechanical ventilation. The patient presented clear signs of heart failure such as pulmonary edema, pulmonary congestion of new onset on the chest X-ray and clear hypoxia. A new TTE and a transesophageal echocardiography (TEE) was performed, revealing normal dimensions of the aortic root, loss of structure of the tricuspid aortic valve with prolapse of the right coronary and non coronary cusp leaflets, causing a massive regurgitation (Figure $1 \& 2$ ). A normal ventricular diameter was observed, with no signs of hypertrophy and left ventricle ejection fraction of $69 \%$. The mitral valve presented a slight insufficiency. These findings plus the patient's history suggested that the mechanism that originated the aortic regurgitation was traumatic. Lesions related to endocarditis were ruled out.

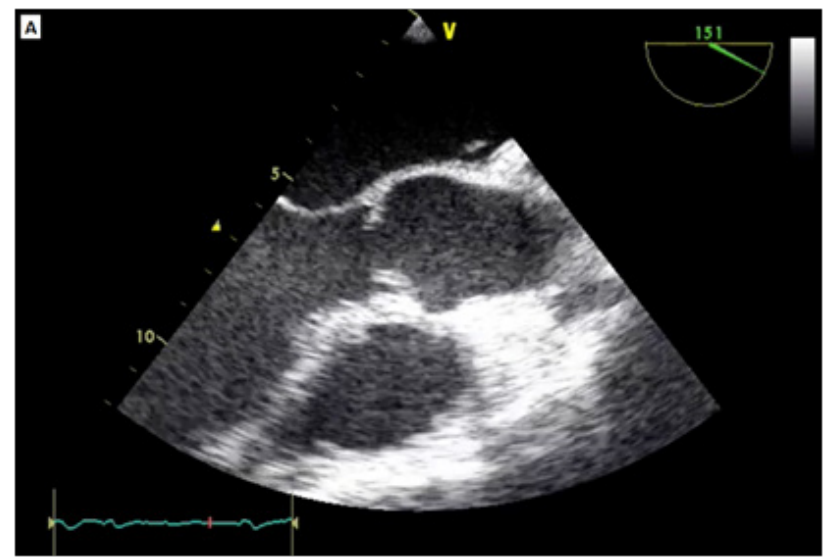

Figure I Transesophageal echography, AV long axis view, lack of coaptation of the aortic valve at a central level in telediastole. 


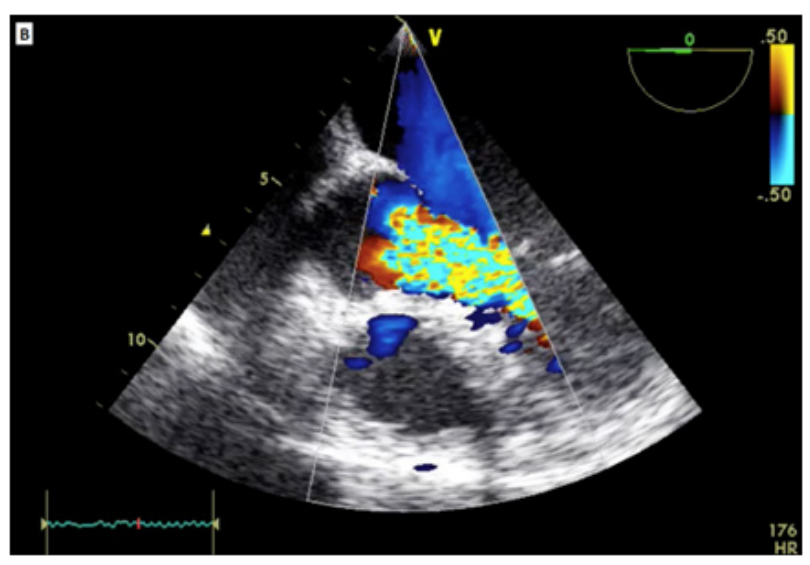

Figure $\mathbf{2}$ Long axis view, jet of massive aortic insufficiency that occupies the complete outflow tract of the left ventricle.

In the operating room, a disinsertion of the commissure between the non-coronary and right coronary cusp was confirmed, causing a prolapse of both thenon-coronary and right coronary leaflets. No endocarditis signs were observed and there was a normal aortic valve structure (except the disinsertion of the commissure) without congenital defects (Figure 3).
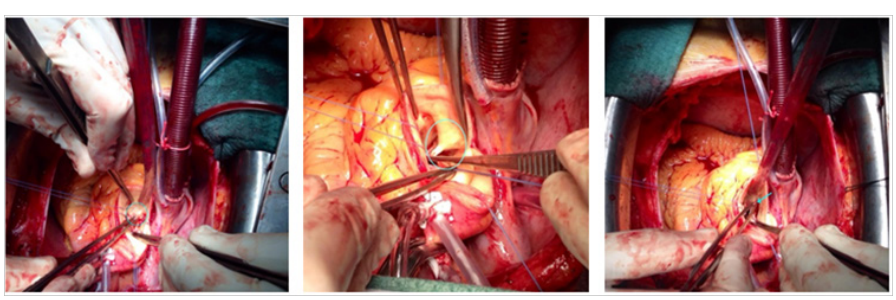

Figure 3 shows the disinsertion of the commissure between the noncoronary and right coronary cusp while the rest of the valvular structure is normal. There were no signs of endocarditis or congenital defects.

The failing aortic valve was replaced with an On-X®mechanical valve \#21 in intra-annular position.

\section{Outcome and follow-up}

Postoperative echocardiography showed a well-functioning prosthetic aortic valve, normal left ventricle size and left ventricular ejection fraction. The patient had an uneventful recovery and was discharged 2 weeks after surgery. On outpatient follow-ups he has shown full recovery.

\section{Discussion}

The clinical spectrum of cardiac injuries after a blunt chest trauma can vary from myocardial contusion to valvular rupture. The most common lesion found in this scenario is myocardial contusion; traumatic valve injury is not as common and usually has a severe and acute presentation $^{5-7}$ although it may have a late onset presentation. ${ }^{8}$ When there is cardiac valve involvement, the aortic valve is most frequently the one affected, followed by the mitral and finally the tricuspid valve. ${ }^{9}$

Post-traumatic aortic valve regurgitation can usually be found with sternal or multiple rib fractures. When there is a fractured of the thoracic wall, the movement of the heart is restricted by the fractured sternum and a force is exerted on the ascending aorta rather than on the aortic isthmus, affecting the column of blood in the aorta, which at the same time could rupture the ascending aorta or a valve apparatus. ${ }^{2}$
The mechanism that leads to traumatic aortic valve regurgitation is a massive increase in the intra-thoracic pressure that leads to an increase in intra-cardiac pressure during a vulnerable phase of the cardiac cycle. For the aortic valve, this happens during the early diastolic phase, which is the phase of lower left ventricular pressure. A high-pressure difference develops across the closed aortic valve and causes aortic valve damage.,10,11 Tear or avulsion from the annulus of one aortic valve cusp is the most frequently observed aortic valve lesion, being the non-coronary cusp the most frequent one $\mathrm{e}^{12}$ the reason is that if the injury occurs while the valve is closed, the overpressure cannot be dampened by coronary flow and causes a tear or disinsertion of the leaflet. ${ }^{13}$

Diagnose of late aortic insufficiency is suggested by heart failure and/or a new diastolic heart in a patient with history of blunt chest trauma, the imaging test of choice is a transesophageal echocardiography, since it can offer higher resolution and real time assessment of both function and structure of the valvular apparatus.

\section{Diferential diagnosis}

The clinical differential diagnosis of weaning induced cardiac failure would typically include left ventricle dysfunction, coronary disease, COPD with hypertension during SBTs (spontaneous breathing tests) and hypertrophic cardiomyopathy. ${ }^{14}$ The patient had no history of COPD or any respiratory symptoms before the accident and the initial TTE had ruled out pulmonary hypertension. Hypertrophic cardiomyopathy was also ruled out in the initial TTE.

The patient had no history of coronary disease; however, if the second TTE would not have revealed the aortic regurgitation, the next step in the differential diagnosis would have been to perform a coronary angiography in order to rule out coronary disease.

\section{Conclusion}

Traumatic regurgitation is a rare complication of blunt chest trauma, and usually the symptoms have a severe and acute presentation.

Late presentation of traumatic aortic regurgitation should be considered in patients with a history of chest trauma even if valvular lesions were initially ruled out.

\section{Conflict of interest}

The author declares no conflict of interest.

\section{Acknowledgments}

None.

\section{Funding}

None.

\section{References}

1. Neschis DG, Scalea TM, Flinn WR, et al. Blunt aortic injury. $N$ Engl $J$ Med. 2008; 359(16):1708-1716.

2. Obadia JF, Tatou E, David M. Aortic valve regurgitation caused byblunt chest injury. Br Heart J. 1995;74(5):545-547.

3. Symbas PJ, Horsley WS, Symbas PN. Rupture of the ascending aorta caused by blunt trauma. Ann Thorac Surg. 1998;66(1):113-117.

4. Giuseppe Bruschi, Salvatore Agati, Fiore Iorio, et al. Papillary muscle rupture and pericardial injuries after blunt chest trauma. European Journal of Cardio-thoracic Surgery. 2001;20(1):200-202. 
5. L Cui, Y Suo, L Han, et al., Traumatic tricuspid regurgitation with ruptured chordae tendinae caused by a punch in the chest, Int J Cardiol. 2015;182:102-104.

6. López-Garrido M, Ruiz-Salas A, Carrasco-Chinchilla F. Late presentation of traumatic aortic regurgitation. Int J Cardiol. 2015;199: 389-390.

7. Kan CD, Yang YJ. Traumatic aortic and mitral valve injury following blunt chest injury with a variable clinical course. Heart. 2005; 91(5):568-570.

8. Matteucci M, Rescigno G, Altamura G, et al. Delayed traumatic aortic cusp detachment mimicking aortic dissection. Ann Thorac Sur. 2006;82(3):1093-1095.

9. Bruschi G, Agati S, Iorio F, et al. Papillary muscle rupture and pericardial injuries after blunt chest trauma. Eur J Cardio thorac Surg. $2001 ; 20: 200-202$.
10. Taguchi $\mathrm{S}$, Ueda $\mathrm{T}$, Inoue $\mathrm{Y}$, et al. Traumatic aortic regurgitation caused by a detached commissure. Gen Thorac Cardiovasc Surg. 2007; $55(1): 32-34$.

11. Li W, Ni Y, Chen X, et al. Aortic valve tear with severe aortic regurgitation following blunt chest trauma. J Cardiothorac Surg. 2011; 6:84.

12. Bernabeu E, Mestres CA, Loma-Osorio P. Acute aortic and mitral valve regurgitation following blunt chest trauma. Interact Cardio Vasc Thorac Surg. 2004;3(1:)198-200.

13. Park SM, Kim DH, Kwak YT, et al. Triple aortic root injury. Ann Thorac Surg. 2009;87(2):621-623.

14. Christina Routsi, Ioannis Stanopoulos, Stelios Kokkoris, et al. Weaning failure of cardiovascular origin: how to suspect, detect and treat-a review of the literature. Routsi et al. Ann Intensive Care. 2019; 9(1):6. 